\title{
Pressure Vessel Fluence Calculations for the Hungarian VVER-440 Units for the Power Uprate and the Llifetime Extension
}

\author{
Gábor Hordósy, György Hegyi, András Keresztúri, Csaba Maráczy, Emese Temesvári ${ }^{1}$, \\ and Éva M. Zsolnay ${ }^{2}$ \\ ${ }^{1}$ Hungarian Academy of Sciences, Centre for Energy Research, P.O.B. 49, 1525 Budapest 114, \\ Hungary \\ ${ }^{2}$ Institute of Nuclear Techniques, Budapest University of Technology and Economics, Múegyetem rkp. \\ 3-9. 1111 Budapest, Hungary
}

\begin{abstract}
A major project was launched at Paks NPP, Hungary, to investigate the possibility of lifetime extension up to 60 years. At the same time, new fuel types with higher enrichment and containing pins with gadolinium have been introduced. Due to these plans, the radiation load of the pressure vessel was evaluated up to 60 years irradiation, taking into account the past and planned future cycles. The computational procedure, elaborated and validated earlier for the fast flux calculation in the pressure vessel was modified for the new fuel types. The neutron source at the core boundaries was taken from core design calculations and the neutron transport from the source to and through the pressure vessel was followed by Monte Carlo calculations. A number of calculations were performed to adequately follow the change of the neutron source. The paper details this procedure, the used Monte Carlo model, the influence of the different reloading schemes on the radiation load and the calculated results.
\end{abstract}

\section{Introduction}

The flux at the pressure vessel may change significantly with time due to the different parameters, like control rod group positions, type of fuel assemblies close to the reactor core periphery, reactor power and reloading schemes. At Paks NPP new fuel types with $4.25 \%$ and $4.7 \%$ enrichment, having fuel pins with gadolinium and different axial height have been introduced recently. This can amplify this change. Consequently, for an accurate prediction of the pressure vessel fluence, the change of the neutron source in the reactor core (named as "core" further on) should be followed with sufficient frequency. To determine the fluence as the function of time (i.e. number of cycles) a number of shielding calculations were performed. To speed up this process, a mixed procedure was developed, validated and applied at KFKI AERI earlier [1]. In this procedure the neutron source was determined on the boundaries of the core from deterministic calculations, and this source is used in the subsequent Monte Carlo calculations performed by MCNP. This way, the neutron transport inside the core can be investigated in details by the KARATE core design code, taking into account the changing of core design and operation parameters.

This is an Open Access article distributed under the terms of the Creative Commons Attribution License 2.0, which permits unrestricted use, distribution, and reproduction in any medium, provided the original work is properly cited. 
Using this method, a detailed 3D fluence distribution can be determined in the pressure vessel for all four units of Paks NPP during reasonable computing time. These calculations were performed assuming different fuel types and reloading strategies until the end of extended lifetime.

\section{Method of the Calculation}

KARATE code system (developed in KFKI AERI) consists of 4 calculation stages (levels). The final "global" level is a 3D nodal diffusion type code which performs a coupled neutron physicalthermohydraulical calculation. The nodes are the fuel assemblies subdivided by the axial layers. It solves the neutron transport equation in linearly anisotropic approximation. i.e. the scalar neutron flux and the net current values are also calculated on the surfaces of the nodes. KARATE was validated against a number of measurements and benchmarks [2], it is routinely used at Paks NPP for an independent checking of the core design. The 4 calculation stages are coupled with a consistent parameterization of the results and via boundary conditions. The 4 levels are as follows.

- 1st level: Multigroup spectral calculations to determine the few-group cross-sections for the further 2- or 4-group calculations and the multi-group energy spectra of the neutron flux and current. 35 thermal and 35 epithermal groups are used.

- 2nd level: 4-group, 2-dimensional fine mesh reflector albedo calculations to evaluate the corereflector albedo matrices

- 3rd level: 2-group, two-dimensional fine-mesh calculations to determine the pin-wise power distribution of the fuel rods.

- 4th level: core-level, nodal calculations, which calculate the power distribution at the assembly level and the boundary conditions for the 3rd level of the calculations.

The surface dependent, multigroup, linearly anisotropic boundary condition can be evaluated as a combination of the 2-group flux and current spectra on the node faces obtained on the 4th level and the multi-group spectra of the 1 st level of the KARATE calculations. The un-normalized multi-group scalar flux and current (1st level) must be normalized, which can be made by using the 2-group scalar fluxes and net currents (4th level). The multigroup currents (calculated by the COLA program) are normalized to the 2-group currents. This way, the currents give account of the geometrical difference of the various faces.

Using the KARATE global module for the pressure vessel source calculations, the assemblies are divided into nodes by several horizontal planes. An axial segment of an outer surface of a fuel assembly in the outermost row of the core is called as "page" in the following when this segment corresponds to a node. These "pages" are numbered according to a scheme, and their surface and the outward normal vector is denoted by $\mathbf{F}_{\mathrm{i}}$ and $\mathbf{n}_{\mathrm{i}}$. From the neutron distribution at the boundary of the core and the reflector depending on the position, energy and flight angle calculated by the KARATE system the source of the Monte Carlo calculations can be determined.

If the angular current density and flux is denoted by $\mathbf{J}^{*}(r, E, \Omega)$ and $\Phi^{*}(r, E, \Omega)$, where $r$ is the position, $E$ is the energy and $\Omega$ is the flight angle, then $\mathbf{J}^{*}(r, E, \Omega)=\Omega \Phi^{*}(r, E, \Omega)$. In linearly anisotropic approximation, the number of neutrons leaving the core across the page $\mathbf{F}_{\mathrm{i}}$ per unit time with energy $E$ and flight angle $\Omega$ is

$$
\mathrm{S}_{\mathbf{i}}(E, \boldsymbol{\Omega})=\int_{\mathbf{F}_{i}} \mathrm{~d} \mathbf{r}\left(\mathbf{n} \mathbf{J}^{*}\right)=\int_{\mathbf{F}_{i}} \mathrm{~d} \mathbf{r}(\mathbf{n} \boldsymbol{\Omega})\left[\frac{1}{4 \pi} \Phi(\boldsymbol{r}, E)+\frac{3}{4 \pi} \mathrm{J}(\mathbf{r}, E)(\mathbf{n} \boldsymbol{\Omega})\right]
$$

where $\boldsymbol{n}$ is the outward surface normal of the page, $\boldsymbol{r}$ is a point on that page and $\boldsymbol{n} \Omega>0$ is assumed. This equation can be written as the sum of two terms with linear and quadratic angular dependence, 


\section{$15^{\text {th }}$ ISRD}

separating the angular dependence from dependence on the energy and on surface in each term:

$$
S_{\mathrm{i}}(E, \boldsymbol{\Omega})=S_{\mathrm{i}, 1}(E)(\mathbf{n} \boldsymbol{\Omega})+S_{\mathrm{i}, 2}(E)(\mathbf{n} \boldsymbol{\Omega})^{2} .
$$

Here the index ${ }_{i}$ refers to dependence on the surface.

Using this result, the source can be defined for each page as a weighted sum of a linearly and a quadratic anisotropic term. The weights depend on the energy and on the selection of the page. The calculation can be done in two steps, one calculation with linearly and one calculation with quadratic anisotropic angular dependence of the source. Using these sources in separate calculations, the space dependence of the energy spectra (i.e. using different spectra on different surfaces) can be included into the MCNP input. Having done the two MCNP calculations with different angular distributions, the weighted sum of the two calculations gives the neutron flux in linearly anisotropic approximation. The weights follow from integration over $\boldsymbol{\Omega}$ for $\boldsymbol{n} \boldsymbol{\Omega}>0$, and their value is $1 / 4$ for scalar flux source and $1 / 2$ for the net current source. The input data needed from KARATE calculations for the MCNP calculations are: the multigroup spectra of the scalar flux and net current for each page.

For practical reasons, the surface source was approximated by source given in thin cells. (If complicated surface source is used, the geometry plots made by MCNP become obscure.) To avoid this difficulty, the source was defined in the water gap between the boundary of the core and liner. This gap was split into cells corresponding the outward boundaries of the KARATE nodes. This way a source cell corresponds to a page. For the source definition sampling of the neutron depending on the position, the energy and the angle should be given:

Position: At first one cell is sampled, which is one axial level of one edge of an assembly. The probability of sampling the cell is proportional to the integral of the energy spectra. Inside the cell the position distribution is uniform.

Energy: In the sampled cell the neutrons are started according to the 70-group energy spectrum belonging to the given cell. Within a group the starting energy is sampled uniformly.

Flight angle: As it was described previously, two calculations are made with different angle dependence. The calculation due to the scalar flux is made by linearly anisotropic angle dependence, the calculation due to the current is made by quadraticaly anisotropic angle dependence.

In the procedure described above, the energy dependence is changing from cell to cell, and the change of angular dependence is taken into account by performing the two calculations. The results of the two calculations should be combined in a suitable way to obtain the real flux for the calculated regions. The Monte Carlo calculations were made by using MCNP5 [3] with continuous energy representation and with the libraries ENDF/B-V and ENDF/B-VI.

Sensitivity analysis was performed for the evaluated fluence values, and it was found that the uncertainty of the calculated fluence at the inner surface of the RPV is approximately $10 \%$ (one standard deviation). This value contains the uncertainties due to the technological uncertainties (geometrical and density data), Monte Carlo calculations and nuclear data. Comparison of the RPV surveillance measurements with the calculations supports this value of the uncertainty.

The code system was validated in the framework of the REDOS project [4] with measurements on the LR-0 experimental reactor, and by comparison with in situ measurements at the RPV surveillance positions in the Paks NPP [5]. In both cases, good agreement was obtained between the calculated and experimental data. The validation procedure using the RPV surveillance measurements of the Paks NPP involved data obtained from 91 irradiated activation detectors from two reactor units. The fast reactions ${ }^{54} \mathrm{Fe}(\mathrm{n}, \mathrm{p}){ }^{54} \mathrm{Mn},{ }^{93} \mathrm{Nb}\left(\mathrm{n}, \mathrm{n}^{\prime}\right){ }^{93 \mathrm{~m}} \mathrm{Nb},{ }^{63} \mathrm{Cu}(\mathrm{n}, \alpha){ }^{60} \mathrm{Co}$ were used at different positions. The deviation between the measured and calculated reaction rate values in all cases was less than that can be explained by the experimental error and the calculation error.

The VVER-440 core consists of 349 hexagonal assemblies, the lattice pitch is $14.7 \mathrm{~cm}$. The assemblies are consisting of 126 fuel rods. The neutron source was evaluated by the KARATE code 


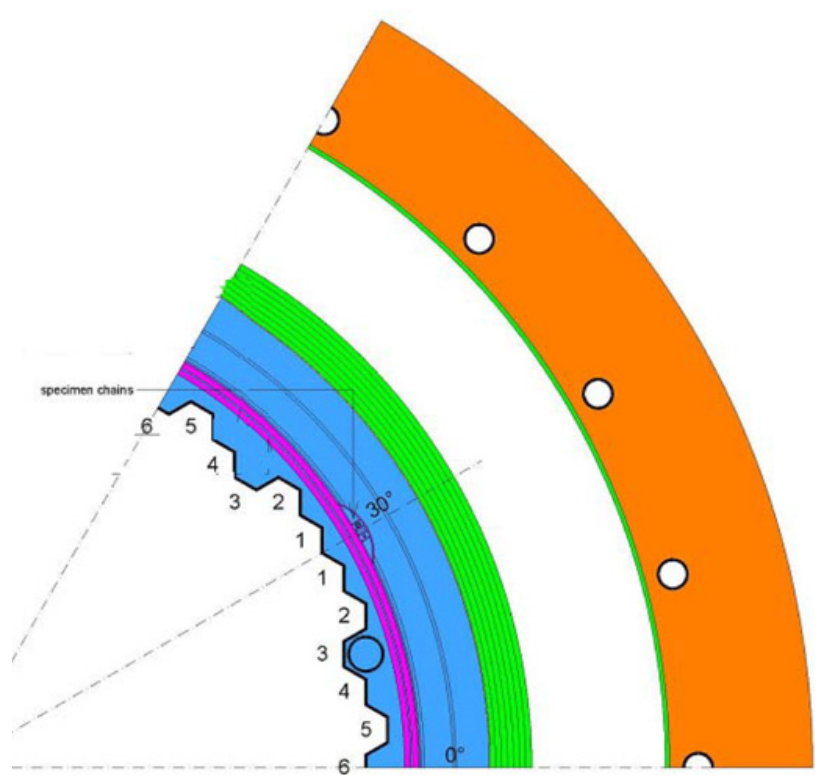

Figure 1. The cross section of MCNP model, numbering the outer assemblies.

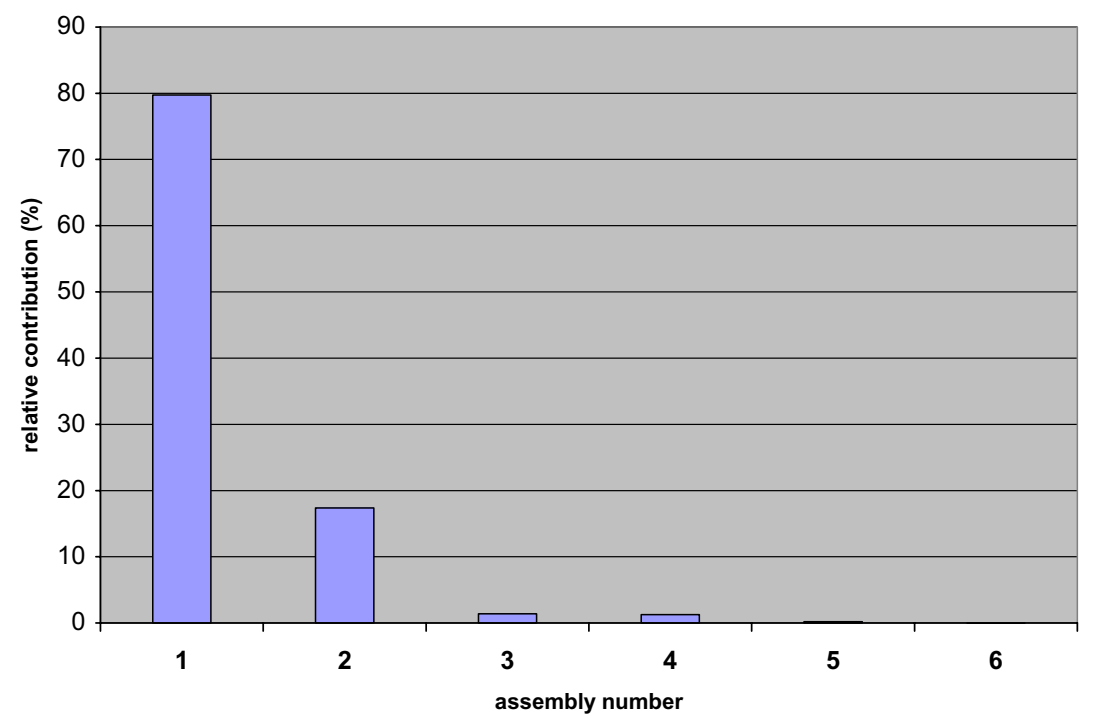

Figure 2. Relative contributions of the assemblies to the pressure vessel fluence at the azimuthal maximum.

on the vertical bounding surfaces of this core. Neutrons leaving the core across the lower and upper horizontal boundaries were neglected.

Originally, 10 axial divisions were used in the KARATE calculations and in the neutron source. However, the new fuel assemblies have different axial height than the previous ones. For accurate representation of a mixed core containing assemblies with different height finer axial division is necessary. This is particularly important for the evaluation of the fluence at the $5 / 6$ weld of the vessel, 


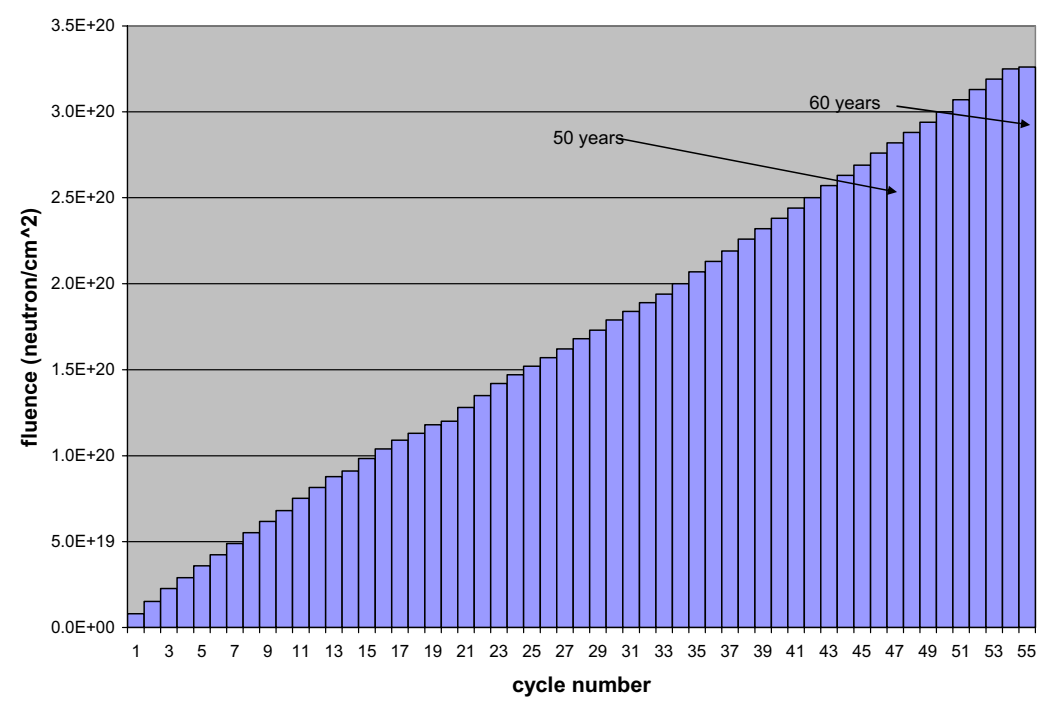

Figure 3. Fluence as function of cycle number at the flux maximum for Unit 2 of Paks NPP.

because its axial position is close to the lower end of the fuel assemblies where the flux changes rapidly in axial direction. In the calculations for the mixed core 41 axial divisions were used.

The Monte Carlo model of the calculation includes the region outside of the core. It extends from the outer surfaces of the core to the outer surfaces of the concrete biological shield surrounding the reactor pressure vessel. The investigated regions are: water, liner, water, core basket, water, core barrel, detector chains, water gap, thermal shield, reactor vessel, cavity and the concrete with the ionization chambers. The calculated area is a 60-degree symmetry sector of the core (see Fig. 1). On the two sides of the 60-degree region reflective boundary condition is applied. On the outer surface of the concrete vacuum boundary condition is given. The material and geometrical characteristics do not change in axial direction in the Monte Carlo model.

The calculated area is extended with $1 \mathrm{~m}$ above and bellow the core height. On the top and bottom of the extended region black boundary condition is prescribed.

Around the core 6 irradiation places can be found, each of them contains 2 RPV surveillance chains, that is series of capsules at different axial positions. A typical chain consists of 19 or 20 capsules. Each capsule contains test specimens and activations detectors. The detectors are several kinds of activation foils. The test specimens are made of reactor vessel materials which are used for the determination of the ductile - brittle transition temperature. The chains can be found next to the core barrel with a stainless steel cover. The position of a pair of chains in the 60 -degree sector of the core can be seen in Fig. 1 . The capsule assemblies with the test specimens and activation foils were modelled in detailed in the MCNP calculations.

\section{Results}

Series of calculations were performed by the above described procedure for the four units of Paks NPP until 50 and 60 years assumed lifetime. For the passed reactor cycles real histories were used, while for the future use of different fuel assemblies and reloading strategies were assumed. It was found that the fuel increment per effective day is varying significantly from cycle to cycle and that this increment depends primarily on the reloading scheme of the given cycle. This can be easily understood examining 


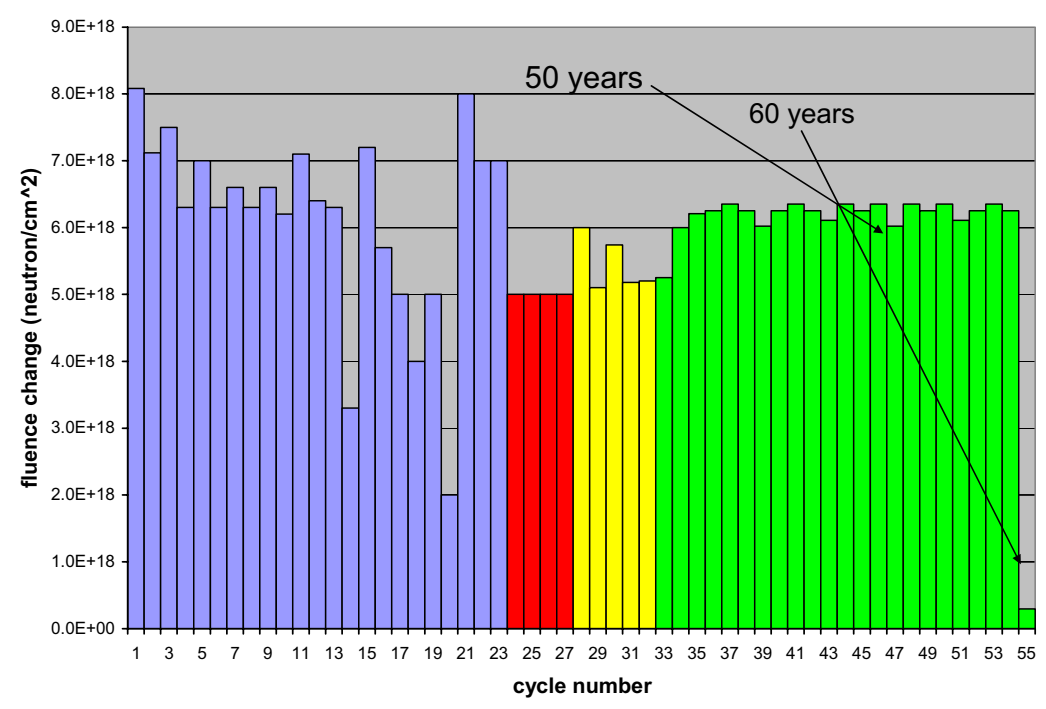

Figure 4. Fluence change per cycle at the flux maximum for the Unit 2 of Paks NPP. Red, yellow and green: uprated power, yellow: $4.25 \%$ enriched fuel is used, green: $4.7 \%$ enriched fuel is used.

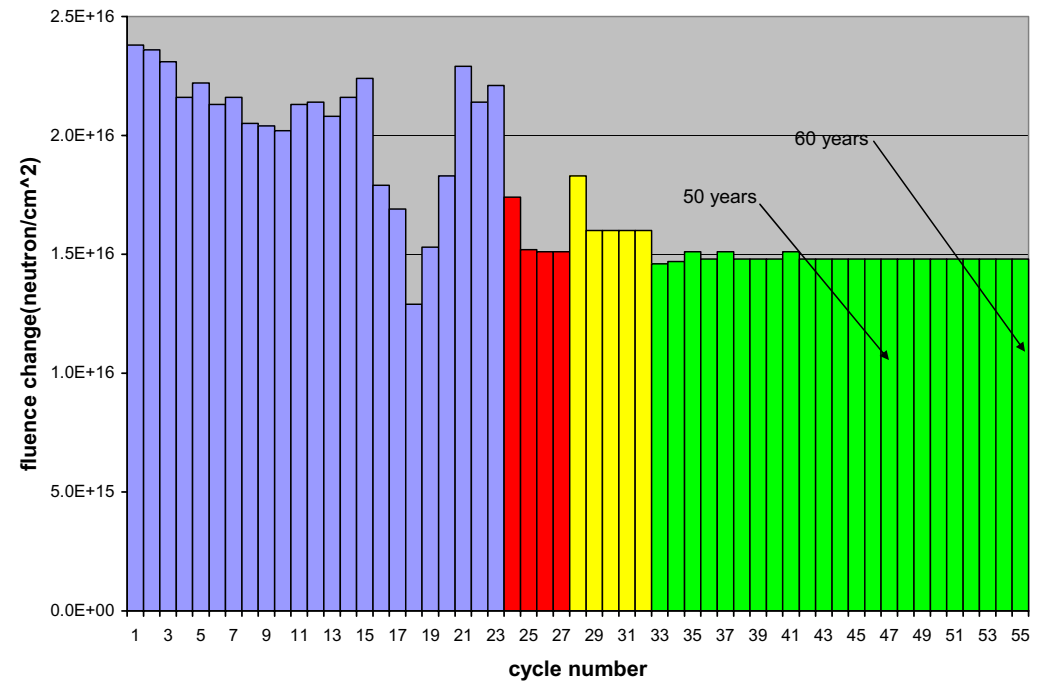

Figure 5. Fluence change per effective day at the flux maximum for the Unit 2 of Paks NPP. Red, yellow and green: uprated power, yellow: $4.25 \%$ enriched fuel is used, green: $4.7 \%$ enriched fuel is used.

the relative contributions of the individual assemblies to the pressure vessel fast flux at the azimuthal maximum, i.e. at 30 degree according to Fig. 1. The relative contributions were determined using the source component flagging option of MCNP. The result is shown on Fig. 2, where the assembly numbering shown on Fig. 1 is used.

It is clear from the Fig. 2 that this flux is determined mostly by the neutron source in the two assemblies closest to the pressure vessel and is determined nearly entirely by four closest assemblies. This way, good core design may decrease the fluence in the future cycles. 


\section{$15^{\text {th }}$ ISRD}

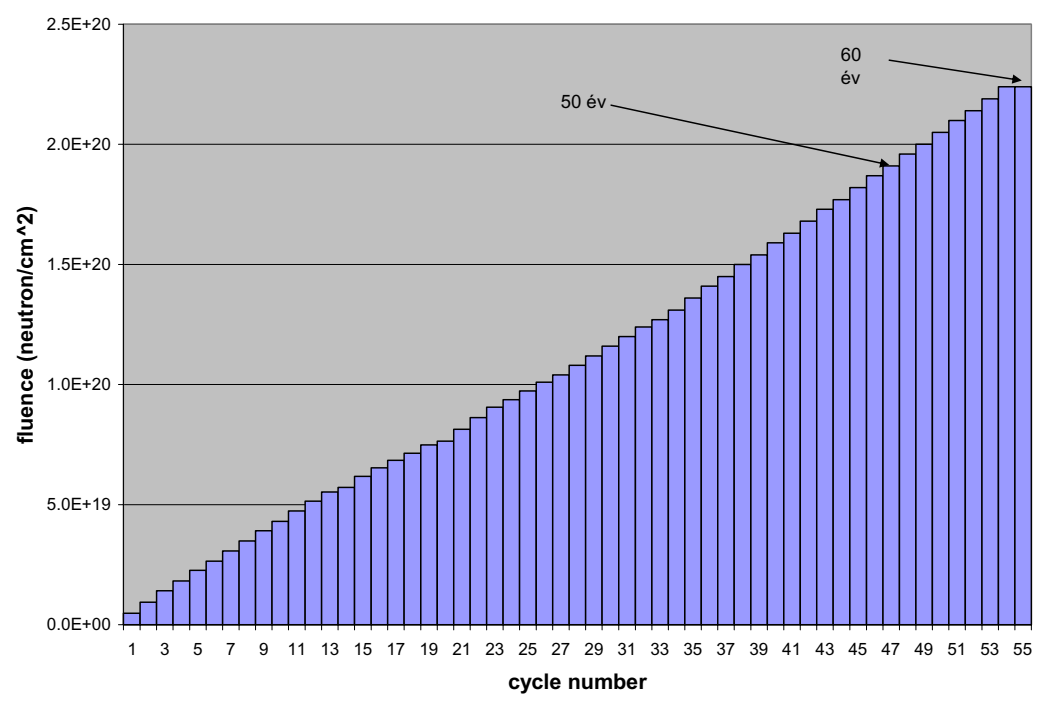

Figure 6. Fluence as function of cycle number at the 5/6 weld (close to the bottom) for Unit 2 of Paks NPP.

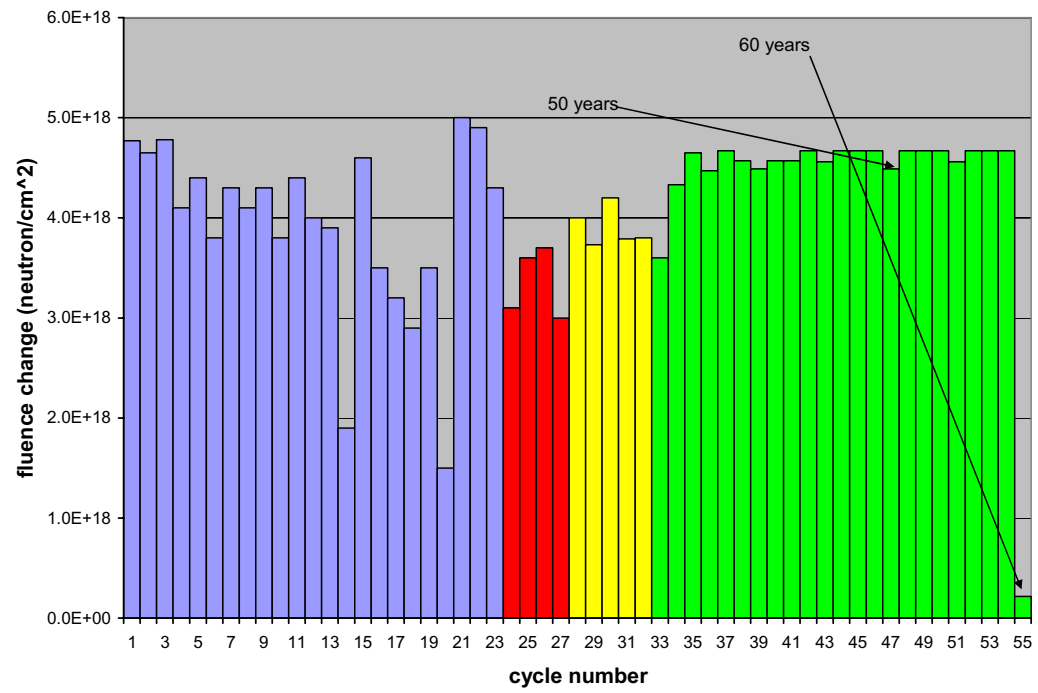

Figure 7. Fluence change per cycle at the $5 / 6$ weld weld (close to the bottom) for the Unit 2 of Paks NPP. Red, yellow and green: uprated power, yellow: $4.25 \%$ enriched fuel is used, green: $4.7 \%$ enriched fuel is used.

Corresponding to this result, we found that the fluence at the end of 50 years lifetime may vary in the range of (2.6-3.6) $10^{20}$ neutron $/ \mathrm{cm}^{2}$, depending on that we assumed the continuation of an early loading pattern or using the recently designed core configuration. Details of fluence change during lifetime are shown on the Figs. 3-8. For the example of Unit 2 when the later option is used for the future. The significant change of fluence increment per cycle is easily observed. In a few cases this is partially due to the short duration of a cycle, but examination of the increment during one full power day clearly shows the importance of reloading strategy (Fig. 5 and Fig. 8). 


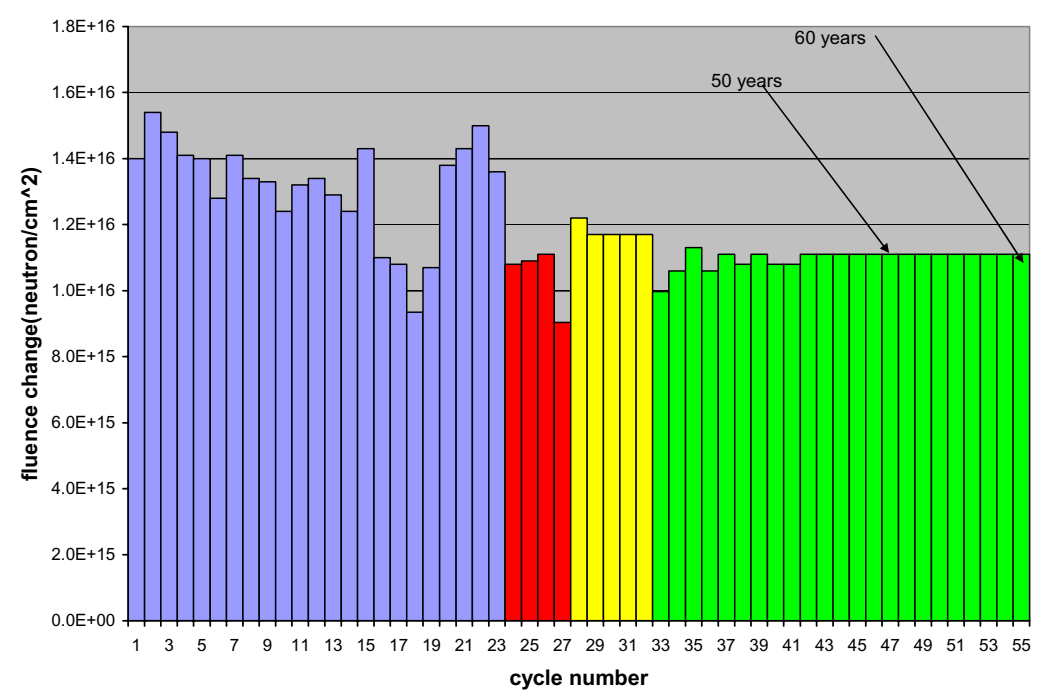

Figure 8. Fluence change per effective day at the 5/6 weld weld (close to the bottom) for the Unit 2 of Paks NPP. Red, yellow and green: uprated power, yellow: $4.25 \%$ enriched fuel is used, green: $4.7 \%$ enriched fuel is used.

\section{Summary}

A coupled deterministic-Monte Carlo computational procedure used for pressure vessel fluence calculations was described. The method was applied for the units of Paks NPP and the fluence until the end of the extended lifetime was evaluated. The influence of the reactor power uprate and of using different fuel types on the fast neutron exposure of the reactor pressure vessel was studied. It was found that the neutron fluence increment can be kept relatively low using appropriate reloading patterns.

\section{References}

[1] G. Hordósy, Gy. Hegyi, G. Hordósy, A. Keresztúri, Cs. Maráczy, E. Temesvári, P. Vértes, E. Zsolnay, "Pressure vessel calculations for VVER-440 reactors", Radiation Protection Dosimetry, 115, Page 100-103, 2005A

[2] A. Kereszturi, Gy. Hegyi, L. Korpas, Cs. Maraczy, M. Makai, M. Telbisz, "General features and validation of the recent KARATE-440 code system", Int. J. of Nuclear Energy Science and Technology, Vol. 5, No. 3, pp. 207-238, URL: http://www.inderscience.com/10.1504/ I JNEST . $2010.033476(2010)$

[3] MCNP - A General Monte Carlo N-Particle Transport Code, Version 5, Volume I: Overview and Theory, LA-UR-03-1987, Volume II: User's Guide, LA-CP-03-0245, Volume III: Devel-oper's Guide, LA-CP-03-0284

[4] B. Boehmer, Ballesteros, J. Konheiser, B. Ošmera, J. Kyncl, G. Hordósy, A., Keresztúri, S. Belousov, K. Ilieva, D. Kirilova, M. Mitev, V. Smutný, E. Polke, S. Zaritsky, C. Töre, P. Ortego: Deep Penetration Benchmarking Conclusions and Recommendations. Redos/R(07)/ 2004/issue 1

[5] Gábor Hordósy, György Hegyi: Reactor Physical Calculations for the Study of the Pressure Vessel Radiation Damage of the Paks Nuclear Power Plant. Report in Hungarian. AEKI-RAL2004/752/02/M2, Budapest 2008 (in Hungarian) 